\title{
Pasado y presente del Archivo histórico de la Provincia de Río Negro
}

\author{
Past and present of the Historical Archive of the province of Río Negro
}

\author{
Cecilia Palma \\ cecilia.palma.24.3@gmail.com \\ Archivo Histórico de la provincia de Río Negro, \\ Argentina \\ Pilar Pérez \\ pperez@unrn.edu.ar \\ Universidad Nacional de Río Negro, Argentina
}

Recepción: 09 Abril 2020

Aprobación: 10 Julio 2020

Publicación: 01 Junio 2021

Cita sugerida: Palma, C. y Pérez, P. (2021). Pasado y presente del Archivo histórico de la Provincia de Río Negro. Aletheia, 11(22), e091. https://doi.org/10.24215/18533701e091
Resumen: Este trabajo busca reconstruir una historia del Archivo Histórico de la Provincia de Río Negro. Esta institución está cumpliendo medio siglo de vida y en su trayectoria se revelan diferentes avatares de la historia rionegrina. El objetivo es evidenciar periodos de funcionamiento del archivo, así como políticas que lo atravesaron y que dan por resultado su presente. Consideramos que los archivos lejos de ser instituciones dadas y estancas reflejan relaciones de poder y disputas políticas en torno a las narrativas históricas y producciones de identidad hegemónicas. Además, y en base al informe producido por la Dirección de Archivo Intermedio del AGN en el 2017, el AHPRN contiene un acervo destacado en la Patagonia. Sin embargo, sus condiciones de acceso y conservación se encuentran en riesgo debido tanto a la carencia de clasificación y ordenamiento como a las inundaciones recurrentes que sufrió entre el 2018-2019. Ante una inminente pérdida de material documental nos preguntamos ¿Cuándo surge el AHPRN y con qué objetivos? ¿Cómo se reunió, organizó y creó este Archivo? $¿$ Qué rol tuvo y tiene como lugar de la memoria? Para reconstruir la vida del Archivo realizamos entrevistas y consultas personales a trabajadores retirados y activos. Recurrimos también a normativa y artículos de prensa. Así como a nuestra propia experiencia en tanto trabajadora y usuarias -respectivamente- del Archivo.

Palabras clave: Archivo Histórico, Río Negro, Políticas de memoria, Estado.

Abstract: This work seeks to reconstruct a history of the Historical Archive of the Province of Río Negro. The fifty years old trajectory of the institution reveals different vicissitudes of Rio Negro's history. The objective of this paper is to organize periods of operation of the archive, as well as the policies that ruled it in order to understand its present. We consider that archives, far from being given and stagnant institutions, reflect power relations and political disputes around historical narratives and hegemonic identity productions. In addition, and based on the report produced by the Argentina's General National Archive in 2017, the HAPRN contains an outstanding collection in Patagonia. However, its access and conservation conditions are at risk due to both the lack of classification and ordering and the recurring floods it suffered between 2018-2019. Faced with an imminent loss of documentary material, we ask 
ourselves when did the HAPRN arise and with what objectives? How was this Archive assembled, organized and created? What role did and does it have as a place of memory? To rebuild the life of the Archive, we conducted interviews and personal consultations with retired and active workers. We also resort to regulations and press articles. As well as our own experience as a worker and users -respectively- of the Archive.

Keywords: Historical Archive, Río Negro province, Politics of memor, State.

\section{INTRODUCCIÓN}

Este trabajo reconstruye una historia del Archivo Histórico de la Provincia de Rio Negro teniendo en cuenta preocupaciones que se derivan de la archivística pero con un especial énfasis en la experiencia diacrónica de conformación de un Archivo. El mismo forma parte del proyecto de investigación "Archivos y narrativas de la Nor-Patagonia” (PI-UNRN 40B731) radicado en la Universidad Nacional de Río Negro. Nos interesa preguntarnos por la decisión de crear un Archivo Histórico, la reunión y selección de sus fondos documentales; la calidad de guarda a lo largo del tiempo; las disposiciones que lo regulan; las características de su depósito entre otros temas atinentes a la disciplina (Heredia Herrera, 1986). Buscamos hacerlo con una fuerte impronta histórica, con el ánimo de conocer el desarrollo de la institución ligado a la construcción identitaria de las y los rionegrinos.

En cierta forma, seguimos la propuesta de pensar el rostro humano del Estado (Bohoslavsky y Soprano, 2010) a partir de conocer las experiencias de trabajadoras y trabajadores del archivo, sus avatares y experiencias. Todo aquello que, en principio, parece información secundaria cuando uno entra a trabajar o bien a consultar un archivo se vuelve insumo para nuestra investigación. Principalmente, porque consideramos que los Archivos históricos jerarquizan y condicionan el ingreso a un tema o problema. A la vez, esta administración que es el Archivo, produce una selección de los acontecimientos que se consideran centrales en el relato histórico y se vuelve performativa (Derrida, 1994).

En este artículo nos proponemos dimensionar, en principio, las políticas oficiales que existieron respecto de la producción y circulación de la memoria histórica dentro de Rio Negro. En segundo lugar, sopesar lo frágil y poroso que resulta la construcción de relatos históricos cuando se indaga en la fundación y sostenimiento a lo largo del tiempo de una de sus fuentes centrales: la documentación de archivo. Esto se vuelve aun más relevante si consideramos que junto con el surgimiento de este Archivo se consolidó un conglomerado de instituciones científicas dentro de la provincia, que fueron exitosas en instalar relatos oficiales y hegemónicos respecto a la historia e identidad rionegrina. Las mismas que -aunque criticadas y en gran parte desacreditadas- hoy se siguen difundiendo desde el turismo, la educación, el relato oficial, la prensa y se encuentran sedimentadas en el sentido común.

En esta disputa siempre política y presente que es la construcción del pasado (Popular Memory Group, 1982) se publicaron y difundieron a lo largo de medio siglo relatos de verdad que resultaron excluyentes de múltiples realidades e identidades sociales de la Provincia. El Archivo Histórico fue en sus inicios y bajo versiones positivistas de la historia, como veremos, la base legitimante para construir estas verdades. Las que hoy, por ejemplo, dan nombre y fechas celebratorias a pueblos y ciudades, o relatan el pasado de los Pueblos Originarios del territorio presente de la Provincia, entre otros. Relatos que legitiman y estratifican la pertenencia y relevancia social dentro de esta comunidad (Trouillot, 1985).

El territorio nacional de Río Negro se conformó una vez concluidas las campañas al desierto (1879-1885), proceso genocida de expropiación y violentamiento de los Pueblos Originarios del sur (Pérez, 2016). El discurso preponderante sostenido para legitimar este proceso era el de confrontar la barbarie por medio de 
una avanzada civilizatoria. La Conquista se constituye de ahí en adelante en un evento estructurante de la sociedad rionegrina (Pérez y Delrio, 2019) organizando la estratificación social. Durante setenta años los territorios nacionales del sur permanecieron subordinados al Poder Ejecutivo Nacional bajo el argumento de minoridad o falta de madurez para gobernarse. El momento de provincialización, a mediados del siglo XX, otorgó a las elites intelectuales y políticas la oportunidad de reafirmar su labor civilizatoria en las décadas pasadas. En este contexto se inscribiría un pasado y se proyectaría una identidad provincial.

Nos centramos en la construcción oficial de la memoria reparando en una de las instituciones que perdura desde entonces hasta el presente, el AHPRN. Partimos de la premisa que los archivos regulan la ley de lo que puede ser dicho en un sistema de enunciados, con un orden y jerarquías particulares (Foucault, 2001). La dimensión diacrónica que exploramos nos permite asegurar que lejos estamos de querer solamente criticar al Archivo con el que contamos hoy por hoy, sino que avanzamos en etnografiar la construcción del mismo (Comaroff y Comaroff, 1992). Con el objetivo de elaborar más preguntas, nos centramos entonces, en la triple función de los archivos, como espacio de investigación, de información para la transparencia del Estado y, fundamentalmente, de derechos (Nazar y Pak Linares, 2007). Por esto, indagamos también en los objetivos cambiantes que ha tenido el archivo ya que en el presente el AHPRN es central para la defensa de derechos de pobladores y comunidades indígenas y también ha restituido historias familiares perdidas o fragmentadas. ${ }^{1}$

En definitiva, nos interesa conocer esta historia para poder dimensionar todas estas problemáticas y para esto trabajamos desde la reconstrucción de memorias respecto del archivo de las y los trabajadores. Aunque también recuperamos algunos datos de normativas - relevantes para nuestras preguntas-y prensa -raramenteocupada de esta temática. Este trabajo no pretende agotar la temática pero si iniciar la indagación de pasados y presentes de archivos en base a las experiencias que emergen de estos, relativizando, si se quiere, la solemnidad que los antecede. Por otra parte, porque consideramos que aunque los Archivos Históricos son la institución de circulación habitual para investigadores, su historia es aún un ejercicio escasamente explorado en nuestro país.

\section{SURGIMIENTO DEL ARCHIVO}

El Archivo Histórico de la Provincia de Río Negro fue creado por medio del Decreto 700 del año 1967. El mismo establece -en estrecha sintonía con la Ley 15930 del Archivo General de la Nación- los fines y funcionamiento de la institución. En los considerandos se especifica que el Archivo Histórico está destinado fundamentalmente a la conservación y acopio de documentos para la investigación histórica, así como cumplir de soporte para la misión de esta:

\footnotetext{
Que la provincia no puede permanecer indiferente ante la realidad que atenta contra la conservación del acervo espiritual de la comunidad; que la investigación histórica no es una mera actividad de erudición, sino un verdadero servicio de alto interés público, que debe contar para ello con fuentes organizadas de información, que la formación de una conciencia integradora de la nacionalidad no es posible sin un conocimiento de los hechos y los móviles que los produjeron, como también su proyección sobre generaciones futuras (Decreto 700/67).
}

Claramente el Archivo Histórico -en función de la historia como servicio público- se legisla como una institución clave de proyección de la Provincia hacia el futuro. Por su parte, el articulado prevé el tipo de documentación a resguardar y la antigüedad mínima de la misma en 30 años (art. 3, 8 y 14); así como las funciones específicas del Archivo (art. 1, 2, 6 y 10); la regulación de acceso y asesoramiento sobre la selección y utilización de la documentación (art. 5, 7, 9 y 15) y la seguridad sobre el material (art. 11, 12 y 13). El Decreto en su conjunto demuestra la puesta en valor del Archivo y le otorga un "guardián" en la creación de un Consejo Honorario de "estudiosos del pasado rionegrino". Este consejo tenía la potestad de asesor sobre dudas y problemas que emergieran de la puesta en funcionamiento del Archivo. 
Tal y como lo plantea Palma (en prensa) la coyuntura histórica de creación del Archivo -entre otras instituciones que describiremos con las que se encuentra intrínsecamente ligado- se corresponde con el proceso tardío de provincialización ${ }^{2}$ y de creación de una identidad rionegrina oficial que se vuelve excluyente y organizadora de la sociedad. Este proyecto estuvo a cargo de una combinación de fuerzas políticas y sociales de Río Negro que se vinculan estrechamente desde la "Conquista del desierto" en adelante. Este "entramado eclesiástico-científico-militar" (Cecchi, 2018) generó un relato y símbolos históricos de trascendencia en la educación y ciudadanización del rionegrino desde la segunda mitad del siglo XX. Entre estos podemos mencionar, por ejemplo, el escudo y el himno de la provincia. Recién en el 2013, estos símbolos fueron modificados por su contenido racista contra los Pueblos Originarios de la Patagonia que conforman una población significativa dentro de la provincia. ${ }^{3}$

Tengamos en cuenta que, aunque la ley 14.408 de provincialización fue promulgada durante los últimos meses del peronismo en 1955, la transición de los territorios del sur se produjo durante la "Revolución Libertadora" (incluso la convención constituyente). A la vez, los sucesivos golpes de Estado impidieron la conclusión de los dos primeros gobiernos electos dentro de la Provincia (en el marco de proscripción del peronismo, fuerza mayoritaria en la época).

Poco tiempo después de efectuado el golpe en 1966, la auto-denominada "Revolución Argentina" creó un nuevo sistema nacional de planeamiento (Decreto 16.964) que organizaba el territorio nacional en ocho regiones, de las cuales la Patagonia era una. Esta regionalización proyectaba a la Patagonia como la nueva reserva de la nación a partir de la implementación de la construcción de represas hidroeléctricas y la explotación de sus recursos naturales, fundamentalmente el petróleo. Este decreto, duramente criticado por avanzar sobre las autonomías provinciales (Camino Vela, 2011, pp. 136-139), preveía inversiones particularmente sobre lo que se organizó como la "región Comahue" (norte de Río Negro y Neuquén). Este proyecto, del cual perduran símbolos como el "indio Comahue" situado en el ingreso a Villa Regina (una estatua que representa un grupo étnico que nunca existió), no perduró más allá de la dictadura. No obstante, incrementó las tensiones -otrora históricas- entre la región del Alto Valle rionegrino y la zona Atlántica (Viedma, San Antonio y Sierra Grande). La zona del Alto Valle era concebida como la región económicamente pujante y Viedma la ciudad más antigua de la provincia (fundada en 1779), sede principal de la Iglesia Católica en la Patagonia y centro de la burocracia nacional rionegrina desde 1878.

La historia de la provincia, hasta entonces publicada sólo por la Iglesia, estaba aún por hacerse. De ese pasado trabajoso y civilizatorio echaba mano el "entramado eclesiástico-científico-militar" (al que le podemos sumar los sectores de poder económico) que buscaba posicionar a Río Negro frente el nuevo poder nacional y defender el lugar de esta dentro del proyecto desarrollista.

\section{ObJetivos (CAMbiantes) DEL ArChivo}

La necesidad de gestar símbolos y escribir relatos del pasado ahora provincial, cristalizó en la creación de instituciones ligadas a la memoria. En 1964, se creó el Instituto de Investigaciones Históricas de Río Negro que presidió el salesiano Raúl A. Entraigas, reconocido artífice del discurso sobre la nueva provincia y autor del himno y otros hitos memorables de Río Negro. ${ }^{4}$ Según el relato de su sobrino nieto -y trabajador del archivo desde 1972 hasta 1990- Jorge Entraigas, a partir de 1967 el gobierno provincial apoyó financieramente las Jornadas de Historia de Río Negro, patrocinando lo que terminó siendo la Junta de Investigaciones y Estudios Históricos de Río Negro que proyectó bianualmente la realización de Jornadas y Congresos. ${ }^{5}$

Existía una voluntad por institucionalizar estas instancias tanto para otorgarles una continuidad a futuro como también para demostrar la capacidad de valorización, gestión y organización de la cultura provincial. Esto último a modo de reacción contra el discurso imperante acerca de la incapacidad por parte de sus 
habitantes de hacerlo, montado sobre todo desde Buenos Aires, durante los setenta años de existencia de los territorios.

En julio de 1967 se realizaron, en Viedma, las Primeras Jornadas de historia rionegrina bajo el impulso del presbítero Raúl Entraigas. A las mismas fueron invitados -según sus vínculos previos- figuras reconocidas del ámbito académico y del conocimiento de la Patagonia. De estas primeras jornadas se resolvió la creación (por decreto) de un Departamento Archivo Histórico y el Departamento Monumentos, Museos y Lugares históricos; y se definió la realización del Primer Congreso de Historia rionegrina en 1968. El mismo tuvo lugar en Bariloche, distante de las tensiones entre el valle y Viedma.

Ese congreso, desarrollado en la ciudad cordillerana, tuvo un correlato en el mes de noviembre del mismo año en Chubut. Como destaca Binder (2015) en su análisis sobre dicho Congreso, los referentes eran interesados en la historia que provenían o bien de la Iglesia, o bien de las Fuerzas Armadas, o bien eran terratenientes y, también, algunos, académicos o aficionados a la historia (ligados a la prensa local). Desde la perspectiva de este autor, el congreso de Chubut se utilizó como vidriera para una elite que se mostraba pronta para el proyecto desarrollista. La que, además, tenía su propia agenda y se reservaba el lugar de dirigencia para la joven provincia.

El Congreso rionegrino, ocurrido unos meses antes, también da cuenta de un proyecto de construir un relato sobre esta nueva provincia. Entre los participantes podemos mencionar a los padres Paesa y Entraigas, al historiador de la Armada -que en ese momento escribía un libro sobre el rol de la fuerza en la Conquista del Desierto: González Lonzieme, y el intelectual de la familia propietaria de La Anónima y terrateniente Braun Menéndez. ${ }^{6}$ Tal y como destaca Cecchi (2018) este conjunto de intelectuales con un marcado carácter nacionalista y conservador, buscaron mostrar una provincia joven pero pujante y, así, reutilizaron el discurso civilizatorio en el cual las instituciones de la Conquista estaban vigentes y no existía lugar para los indígenas, más que en el pasado. El Archivo Histórico sería la institución creada para certificar la historia que desde los mismos se impulsaba.

De la misma manera, Neuquén había creado en 1965 la Dirección Provincial de Museos, Monumentos y Archivos Históricos. Esta Dirección fue propuesta por la Junta de Estudios Históricos que cumplió un rol fundamental en la definición de la identidad neuquina que sirvió de base fundante a su vez, del Movimiento Popular Neuquino (García, 2006) y que poco tiempo después también efectuó un primer Congreso de historia neuquina.

Esta primera etapa fundacional fue seguida, según el relato del primer director del Archivo, Nilo Fulvi, por una segunda en donde los entrevistados resaltan el impulso del militar y gobernador -de facto- Roberto Requeijo. Este se caracterizó por haber logrado un apoyo importante entre los rionegrinos, al punto de haber armado una fuerza política propia que logró desplazar al radicalismo en las elecciones de 1973, el Partido Provincial Rionegrino (PPR). En 1970, Requeijo creó por decreto $\mathrm{N}^{\circ}$ 104/70 el Centro de Investigaciones Científicas (CIC) que comenzó sus actividades en el ex edificio Municipal de Viedma, dirigido por el controversial Dr. Rodolfo Casamiquela. El CIC buscaba "promover, desarrollar y orientar investigaciones científicas en el ámbito de la provincia". Para ello, destinó un programa de "búsqueda científica" enmarcando estudios sociales y naturales, explorando y ligando las distintas regiones provinciales.

En palabras de Fulvi, se pretendía que el CIC fuera "un generador de información para el planeamiento y el desarrollo. No había ninguna investigación en el aire. Por esto no había ciencia dura". ${ }^{7}$ Aunque se lamenta que muchas veces las investigaciones no se aplicaban a las políticas, sí fueron influyentes y respondieron a algunas necesidades político económicas. ${ }^{8}$

Cabe aclarar que es complicado abordar los alcances del CIC a partir de su fondo documental. Aunque fuera la institución responsable del ingreso patrimonial de objetos y documentación de la provincia, nos encontramos - paradójicamente- con la falta de registros unificados o sistematizados. Se observa un movimiento constante en la constitución de acervos, en la trayectoria de empleados y referentes del CIC (Palma, en prensa). En las historias contadas por empleados de todos los tiempos, es una constante la 
desaparición de biblioratos, cajas y archivos administrativos tras el cierre de las distintas gestiones. Si bien muchas veces consta que fueron devueltos, el fondo documental del CIC es una construcción que aún no se encuentra organizada, involucra a más de una persona y más de mil anécdotas.

Con esta advertencia, podemos sugerir sobre el funcionamiento del CIC tres momentos definidos en función de su organización, políticas de funcionamiento, personal involucrado en sus tareas y fundamentalmente alcance y variedad de las publicaciones propias. Estos son: 1969-1973; 1973-1983; y, finalmente, 1983-1994, cerrando el período con su última publicación. Podemos aproximar un número de 70 empleados en el CIC-DER (Dirección de Estudios Rionegrinos) entre 1969-1994; número que incluye administrativos, dibujantes, investigadores, maestranzas, etc. A esto hay que sumarle las relaciones mediante convenios y becas, que aún no han sido sistematizadas (Palma, en prensa).

En la primera etapa, 1969-1973, el CIC acompañó las políticas desarrollistas provinciales y operó como el respaldo científico de la investigación histórica (con los referentes y perfiles de la historia que ya mencionamos) de las diferentes regiones del ex territorio nacional. La principal estrategia de crecimiento para el CIC durante estos primeros años fue mediante la asistencia y organización de eventos científicos, a partir de los cuales solicitaba y recibía currículums y proyectos de interesados en la zona, a la vez que contrataba o vehiculizaba sus investigaciones, según fuera el caso. De esta manera, llegaron jóvenes profesionales de diferentes universidades del país, para así profundizar estudios sobre Río Negro. Muchos de los investigadores de entonces tenían sus propias líneas de financiamiento, aunque siempre estaba abierta la posibilidad de establecer una relación de estudio y trabajo más estable, lo que sucedió fundamentalmente con profesionales de las ciencias sociales. El régimen de becas, asignaciones y adscripciones, parece haber sido crucial en estos años.

El CIC tenía para 1975, nueve departamentos: 1) Sociales; 2) Ciencias. Naturales; 3) Museos, Monumentos y Lugares Históricos; 4) Archivo Histórico e Investigaciones Históricas; 5) Artesanías Folclóricas; 6) Ciencias de la Tierra; 7) Ciencias Biológicas; 8) Antropología y Folclore y 9) Técnico y de Administración. Incluso había crecido con la incorporación de becarios, lo cual permitía comparar el desarrollo del CIC con el CONICET y sus investigadores de planta formados y becarios en formación. A su vez, el CIC fue desde este período de enorme influencia en la educación rionegrina hasta el presente. Entre otras razones por la producción de manuales y libros de historia de distribución (voluminosa) en las escuelas y bibliotecas de la provincia.

Sin embargo, durante la última dictadura la persecución a varios de sus trabajadores, operada por su principal referente, Rodolfo Casamiquela, desmanteló en gran medida la estructura y los objetivos del CIC en su conjunto. Según Graciela Suárez, Casamiquela "vino a intervenir el CIC, y lo había dicho cuando asumió, que venía a hacer limpieza" (en Cecchi, 2018, p. 12). Si bien la estructura de investigación fue atacada, en el decir de Fulvi "al museo y al Archivo no los pudieron desaparecer". Aun así, existe en la memoria social el registro de que en ese periodo muchos documentos fueron sustraídos y nunca retornados al Archivo.?

El retorno democrático fue, en una apreciación común de los entrevistados, una enorme desilusión en relación al Archivo. Las políticas del gobernador radical Álvarez Guerrero intentaron descentrar la Secretaría de Ciencia y Técnica "nombrando un referente en Bariloche" ${ }^{10}$. Sin perder de vista las disputas regionales propias de la provincia, esto significaba demasiado lejos -geográficamente- del AHPRN y -concretamentecon poco interés en el mismo. Como destacáramos la tensión de fuerzas políticas de la provincia pasa por la competencia entre el alto valle (Roca-Cipolletti) y Viedma. Bariloche, a pesar de ser la ciudad con mayor población de la provincia, solía quedar por fuera de esta competencia. De hecho, de la Administración de Parques Nacionales o Escuela Militar de Montaña -entre otras instituciones- no queda ningún registro dentro del Archivo.

$\mathrm{Al}$ interior del CIC, la democracia desestructuró pilares de su funcionamiento, principalmente lo desvinculó de Planeamiento y lo desfinanció. Para los entrevistados -Fulvi, Entraigas y Moldes ${ }^{11}$-, representó una "devaluación" el paso a constituirse como "Dirección de Estudios Rionegrinos" que fundamentalmente 
vació de personal, acabó con las publicaciones y desfinanció las campañas. Por otra parte, si bien desde el regreso de la democracia, se buscó modernizar otras áreas de documentación como Catastro o Rentas (a partir de financiamientos del BID), no fue el caso del Archivo Histórico.

Cuando Rebeca Pajón, profesora de historia y trabajadora del AHPRN entre 1995-2015, ingresó a trabajar, el Archivo se encontraba en estado de abandono. Sus gestiones como trabajadora apuntaron primero a limpiar y ordenar el material, con escasas herramientas, ninguna formación y, prácticamente, sin personal. Sin embargo, al trabajo de ordenamiento y conservación básicos de las primeras etapas, se sumaron nuevos usuarios al Archivo. Dejó de ser pensado y utilizado únicamente para la investigación y comenzó a atender pedidos de múltiples dependencias estatales (Policía, Dirección de tierras, Justicia, CODECI, ${ }^{12}$ entre otras); gradualmente también, comenzó a responder a demandas de la ciudadanía en general.

A pesar de la ampliación hacia una función social del Archivo, las políticas estatales de los ochenta y noventas continuaron relegando esta institución en el vértice constante de sus cambiantes organigramas. Así, pasó por diversas dependencias. Entre ellas Ministerio de Planificación, de Educación, de Coordinación, de Desarrollo Social y, por último, al de Turismo, Cultura y Deportes del que depende en el presente.

El cuidado y la preservación del material quedaron muchas veces en manos del criterio de los empleados. Así, relata Pajón que en una oportunidad en que la mayoría de los trabajadores habían tomado vacaciones, desapareció un expediente de alta conflictividad en lo que respecta a la transferencia de tierras rurales en la provincia de Río Negro: el expediente del caso Lewis. ${ }^{13}$ Por esto, relata Rebeca que ella decidió endurecer la política de retiro de expedientes del Archivo (nada más que haciendo cumplir la mencionada Ley 700/67). Esto provocó la intervención de la justicia, que envió personal policial a decomisar los expedientes de Tierras. Pajón lo relata en primera persona: "No, no se los voy a permitir, los expedientes no salen de acá... venga que le muestro el fondo Tierras... ¡Cuando vio la cantidad! Creerían que era un paquetito! [risas]". ${ }^{14}$ Allí quedó la disputa por el fondo que sigue estando en guarda legal del AHPRN gracias y a partir de las gestiones formalizadas posteriormente por esta trabajadora.

El AHPRN es desde las primeras décadas del siglo XXI un garante indiscutido de derechos para pobladores -sobre todo mapuche y tehuelche- a quienes se les negó históricamente el reconocimiento de las tierras que ocupaban. Aun así, su ocupación quedó registrada en documentación incluso marginada dentro del propio Archivo (sin organizar, ni clasificar). Al presente, estos expedientes e inspecciones de tierras, por nombrar algunos fondos, hacen visibles movimientos en el territorio, así como permiten reconstruir la dinámica de expropiación de tierras a lo largo del siglo XX, y devolverle trayectorias a familias que se encontraron forzadas a migrar por desalojos y nos permite conocer estrategias de organización política silenciadas o relegadas (Pérez, 2016, Dall'Armellina y Palma 2018 y Kropff et al, 2019).

\section{El Archivo DesDe Su PERSONAL}

En los tiempos de organización inicial del Archivo, a fines de los años sesenta, los responsables si bien sabían que era necesario tener "fuentes organizadas de información” (Decreto 700/67), no tenían ninguna formación en archivología. Por esto, buscaron capacitarse contactando al Dr. Aurelio Tanodi, de la Universidad de Córdoba, quien dictó un seminario de formación general. También recurrieron al Archivo General de la Nación, y en correspondencia con su orientación hacia la investigación que (re)construyera la identidad provincial, a la Academia Nacional de la Historia. ${ }^{15}$ Fulvi tenía como formación de base el profesorado de historia que se dictaba en Viedma. Mientras que sus compañeras/os eran, con la excepción de Jorge Entraigas, o bien estudiantes o idóneos.

No obstante, la intención era formar profesionales que pudieran desarrollarse dentro del archivo. Aun así, en la última dictadura, la persecución al personal habilitado por el decreto que ordenaba identificar a los empleados "potencialmente disolventes" persiguió y expulsó en exilios internos y externos a parte de los 
trabajadores de esta área de trabajo. Fulvi narra cómo a Victoriano Schillizzi "se lo llevaron de las pestañas delante de mí”. Gracias a las denuncias públicas realizadas por Graciela Suárez, historiadora y trabajadora del archivo en ese período, en el 2008 sabemos que fue el director del CIC, el mencionado Rodolfo Casamiquela, quien se ocupó de "marcar" (identificar) personas en el ámbito laboral. Como en otros aspectos, el terrorismo de Estado disciplinó y desarticuló el proceso en marcha del Archivo. Proceso que pretendía ampliar su clasificación para mejorar el acceso.

Sin embargo, el regreso de la democracia no trajo una recomposición de estas instituciones como era esperado. Las causas deben observarse en los quiebres y continuidades que tiene el periodo posdictatorial. No sin conflicto, la dirección del CIC -dentro de la cual estaba el Archivo- se la dieron al mismo Schillizzi. Sin embargo, al decir de varios de nuestros entrevistados, sus actos como director no eran claros y versaba entre los problemas personales y la conflictividad que (se) generaba habitualmente con sus compañeros de trabajo. Algunos de los cuales habían continuado trabajando desde la dictadura.

Cuando Rebeca Pajón ingresó a mediados de los noventa, la persona que más sabía sobre la documentación albergada en el depósito del archivo era Ramón Varela. Durante los años que trabajó allí, a Ramón le decían el "alma" del Archivo. ${ }^{16}$ Se desempeñaba como maestranza y, aunque no tenía escolaridad, era la persona que sabía cuales eran los fondos, qué expedientes y nomenclaturas tenían, qué años abarcaban. Pero fundamentalmente, Ramón sabía dónde estaban los lugares físicos dentro del vasto depósito de papeles. Con este aliado dentro del Archivo, Rebeca comenzó su formación autodidacta. Por esto, por más de veinte años quienes la conocimos suponíamos que era la directora del Archivo. Aunque este cargo estuvo acéfalo durante la mayoría de los años que ella trabajó allí.

El Archivo también se convirtió desde aquel momento, como sabemos por otras experiencias, en la dependencia a donde se derivan personas complicadas políticamente, como a personas con problemas de salud mental o laboral. Desde su creación hasta 1994, el archivo contó con un promedio de tres trabajadores (aunque con recambios, el número se mantuvo estable). Por el Archivo pasaron un sinfín de empleados que desconocían el trabajo concreto a realizar, que no tenían formación ni se preocupaban por tenerla, junto con personas con mayor interés que valoraban el acervo custodiado en el archivo, y ordenaron, clasificaron y limpiaron el acervo que continúa resistiendo. En esta precariedad laboral, quienes llegaban y se comprometían, muchas veces no perduraban. La formación de este último personal redundó en capacitaciones esporádicas dictadas sobre archivos administrativos y, especialmente, guiadas por el interés personal y los contactos entre trabajadores de otras dependencias, otros archivos y el intercambio con los mismos.

\section{LOS FONDOS DOCUMENTALES}

Tal como referíamos al inicio de este trabajo, el AGN DAI destacó en un informe realizado en el 2017, el volumen y variedad del acervo documental que alberga el AHPRN. Por esto, nos interesa identificar y describir cuáles fueron los mecanismos de acumulación de los diferentes fondos documentales que componen en el presente el archivo. Para esto, son fundamentales los relatos de quienes fueron los principales acopiadores de este material en sus gestiones como trabajadores del archivo.

Nilo Fulvi narra el traspaso del Archivo General guardado en Viedma, de la Gobernación del territorio de Río Negro, en los últimos tiempos previo a la provincialización, a la creación del Archivo Histórico. Dice Fulvi:

Me entero que estaban sacando papeles de la Gobernación, del Archivo General, porque necesitaban espacio. Los cargaban en camionetas, los mandaban a la estación del ferrocarril para venderlos como papel viejo. Y se llevaron algunos dicen 2 o 3 camionetas, hasta que yo me enteré. Fui a mirar y estaban tirando desde el primer piso al patio de la Jefatura estos papeles para después cargarlos en la camioneta. 
Esta primera acción dio por resultado el ingreso de los fondos vinculados a Gobernación del Territorio (1884-1950), incluyendo el período de la Gobernación de la Patagonia (1878-1884); el principio del fondo Policía y parte de Justicia Letrada. Una vez ubicados estos fondos como material patrimonializado entonces como histórico, el Archivo comenzó una segunda e inmediata etapa, la gestión de nuevos fondos.

En este proceso se produjo el acuerdo con la Dirección de Tierras para la administración y guarda de inspecciones y expedientes, que constituye uno de los fondos más extensos del archivo. También se realizó una gestión en la Justicia Federal que permitió el acopio del material de expurgo de la justicia provincial. Llegaron así los materiales de la Justicia Letrada del Territorio en Bariloche y General Roca. Dentro de este primer período, casi como política de rescate de continuas inundaciones, se sumó el fondo del Municipio de Viedma.

A su vez, se constituyó dentro del Archivo una Hemeroteca que contiene diarios y periódicos existentes durante el periodo del Territorio. Este acervo se fue completando paulatinamente a lo largo del tiempo con donaciones puntuales de colecciones de algunos diarios, incluso del período provincial.

El trabajo sobre los fondos en el sentido archivístico fue prácticamente nulo. No hubo más clasificación de los mismos que la que traían al ingresar, intentando mantener el orden con que ingresaban. La mayoría de las veces, los fondos entraron sólo con una lista. Cuando se ha intervenido esta organización, o planteado iniciar alguna, ha sido una división vagamente temática y vinculada a los intereses particulares de los investigadores que trabajaron los fondos. Por esto, la mayoría de los documentos transitaron casi sin modificación en paquetes, tal y como fueran preservados por las instituciones productoras de los mismos. Las tablas de conversión que llama la archivística, a los pasos y protocolos para reorganizar los documentos, descansaban y se creaban en las memorias de algunos trabajadores.

Durante los noventa se realizó el traspaso del resto del fondo vinculado a Justicia Letrada (poder judicial del periodo de Territorios Nacionales). Pajón relata cómo se "enteraron" -una estrategia habitual de acopio de fondos- de que la Justicia estaba por deshacerse de material histórico. Las gestiones desde el Archivo en este período, que invocaron a su vez gestiones anteriores vinculadas al material de justicia, dieron por resultado el acopio de este fondo central y fundamental que son los expedientes de la Justicia Letrada del territorio. ${ }^{17}$

Esta situación de salir al rescate cuando se enteraban de "que iban a tirar papeles" resultó crucial no solo para adquirir nuevos fondos, sino también para que el archivo fuera conocido y de ahí en adelante convocado cada vez que alguna dependencia estatal deseaba hacer lugar.

En esta articulación que devino una verdadera técnica de adquisición de fondos se sumó el fondo “prontuarios policiales”. Dice Pajón:

Cuando la Policía estaba en la gobernación fui al archivo. Era un archivo medieval. Vos entrabas ahí y era como una torre así [señala hacia arriba], toda hecha de cantonera... una cosa rara, rarísima. Vos decías... acá se prende un fósforo y se mueren todos. Mirabas para arriba y se hacía como una pirámide, era incré́ble. (...) Aparece un milico a decirme que iban a mandarme documentación. "Pará”, le digo, “¿Qué documentación? ¿Cuánta documentación? ¿Por qué? ¿La tienen organizada, registrada? ¿Qué mandan y qué no?” Porque, después, sos culpable vos. Vas aprendiendo la cuestión legal para poderte defender. Pusieron los miliquitos a trabajar meses y meses, y entonces ahí hicimos un acta.

Estos prontuarios traían una codificación propia del uso policial que hubo que comprender, de nuevo, a partir de vínculos personales con personal policial que pudiera hacerlo comprensible. Detrás de este ingreso de fondos policiales, llegaron otros que se encontraban en el Penal 12 que pertenecían a comisarías varias del territorio. De todas maneras, hubo fondos que se perdieron aun cuando los trabajadores del Archivo se movilizaron para conseguirlos, como por ejemplo, aquellos pertenecientes al Banco Río Negro. Las implicancias político económicas para el Estado provincial explican por qué este fondo no fue puesto a disposición para la consulta pública. 


\section{El espacio Del ARCHIVo}

Una vez que el archivo fue literalmente revoleado y expulsado de la Jefatura de Policía en 1968, el lugar físico que ocupó fueron las instalaciones de la actual Municipalidad de Viedma. El depósito se situó en el edificio donde funcionaba el CIC y el museo, específicamente en lo que habían sido las antiguas caballerizas de la primera Municipalidad del territorio. Un espacio húmedo y difícilmente acorde a las necesidades de conservación del papel. Sin embargo, el archivo estuvo allí por más de tres décadas.

El espacio destinado para consultas era compartido con la Biblioteca Histórica. La misma fue creada conjuntamente con el AHPRN, como parte del proyecto de producir y desarrollar investigaciones. La Biblioteca Histórica se conformó, según relata Fulvi, por medio de tres entradas de material. Primero, los canjes producidos en los contactos de los investigadores propios de la profesión. En segundo lugar, la donación de libros. Finalmente, por medio de la compra de bibliotecas completas de intelectuales (entre ellos, Hilarión Lenzi y Liborio Justo). ${ }^{18}$

El Archivo fundamentalmente, juntaba tierra y mugre; ya que tanto las personas como el patrimonio estaban sometidas a condiciones para nada habitables. Además, acumulaba hongos, ácaros y otros seres de la fauna local. Al decir de Moldes "el sótano/depósito se inundaba hasta el '91 que me fui... sin gas, sin luz y con gamexane". ${ }^{19}$

Pajón narra una experiencia que catapultó a la opinión pública la situación de abandono y disparó la necesidad de reparar en políticas de conservación del archivo:

Llega Raone ${ }^{20}$ con un traje blanco... Él quería un expediente de Tierras. Los paquetes de Tierras eran grandes, de 40 o 50 expedientes cada paquete. Y había que buscarlo en una escalerita, y desde ahí, arriba en una estantería, sacarlo... que, si se te caía, eso era un caos jera pesadísimo! Ese trabajo lo hacíamos con Ramón. Y cuando viene este hombre, me sigue, bajan el paquete y cae... ¡ Una tierra!....

A partir de entonces, Ramón y Rebeca tomaron la práctica de poner los expedientes al sol para evitar la propagación de los hongos.

A partir del año 2004, la Municipalidad de Viedma solicitó la devolución del edificio donde funcionaban el AHPRN, el Museo Tello, el área de Arqueología y Palentología de la Provincia, y la Biblioteca Histórica. El AHPRN comenzó a mudarse en el 2006 al depósito que tiene en el presente en un edificio no propio, pero perteneciente a otra dependencia provincial. En 2010, el desalojo forzado por el intendente Jorge Ferreyra fue inminente para el resto de las instituciones vinculadas.

En la mudanza a esta nueva dependencia, y frente al reclamo de los trabajadores, se consiguieron estanterías para acomodar los fondos documentales. En el mismo proceso y producto de las gestiones entre trabajadores del Archivo y de la Dirección de Tierras se adquirieron cajas de cartón que sobraban en una dependencia y resultaban útiles en la otra. Así muchos de los paquetes fueron organizados en cajas para garantizar su conservación por primera vez. El depósito actual es de 25 por $25 \mathrm{mts}$., cuenta con oficina para el personal y sala de consulta. Aun así, el edificio pertenece a la obra social de la provincia IPROSS y en el último año la gestión provincial le cedió 6 × 25 mts.

Así como Fulvi remarca que su intención principal fue darle un sentido y carácter histórico a los fondos documentales rescatados de la venta, Pajón destaca que su principal objetivo fue conservar. Esto significa que en cuarenta años el Archivo no avanzó prácticamente en nada a nivel de clasificación y de volver accesible a la consulta el material. Los empleados han mantenido las listas y el orden, lo que no es un esfuerzo menor. Más aun, en los últimos años incluso la cantidad, variedad y conservación de los fondos fue puesta en duda. Entre el 2018 y 2019 el Archivo Histórico sufrió tres inundaciones por negligencias que eran evitables. El material se encuentra aún deteriorado y en riesgo de pérdida.

Sin embargo, en los últimos años se conformó (entre otras organizaciones) la "Asociación Civil por el Patrimonio Histórico Rionegrino” (2017 en adelante) que busca visibilizar y defender el Archivo, anclando 
sus reclamos en la necesidad de existencia del mismo como garante de derechos. En la sinergia entre académicos, trabajadores y la Asociación se encuentra por lo menos instalado a nivel mediático el tema y la valorización del Archivo Histórico. También podemos mencionar algunas propuestas para legislar sobre el Archivo (actualizando el decreto 700/67) que no obstante tienen muy poco impacto en la realidad del mismo. ${ }^{21}$

\section{Conclusiones}

No podemos pensar los Archivos y su contenido sin pensar en su historia y principalmente a partir de las trayectorias de las personas que los habitaron. De esta forma, también comenzamos a analizar la construcción histórica del Estado provincial rionegrino, tema aún escasamente abordado en la historiografía. En la periodización aparecen marcadas políticas de Estado que favorecieron e impulsaron la guarda de la documentación con objetivos concretos y diferentes. Así como otras que claramente abandonaron la intención de resguardar o utilizar esta memoria social e institucional. Finalmente, otras que directamente atacaron la existencia misma del Archivo.

Entre las causas de abandono, nuestros entrevistados acuerdan que uno de los problemas es la legislación que existe. Ya que la misma es insuficiente o inadecuada (fundamentalmente porque son leyes que emulan los usos de archivos administrativos, vaciados del carácter histórico y de derecho). En el caso del Archivo Histórico, los empleados desde sus inicios le dieron sentido y política a la institución. Otra de las razones que se destaca es el lugar cada vez más marginal que fue teniendo en el organigrama de gobierno. En un comienzo claramente se lo visualizaba como una institución fundante para el desarrollo del discurso oficial provincial y legitimante de un sector de poder. Hasta 1983 esta impronta perduró en el Archivo y los discursos académicos y educativos que emergieron de publicaciones ligadas al mismo fueron la base de la producción identitaria y de inclusión/exclusión de Río Negro. A lo largo del tiempo este objetivo fue perdiendo importancia y a su vez el AHPRN también.

Sin embargo, en los últimos años el objetivo y funciones del AHPRN fueron diversificándose. Las demandas sociales han ido modificando la valorización del mismo y hoy incluso se vuelve central en demandas por derechos de aquellos que fueron sus principales excluidos, los Pueblos Originarios.

Por otra parte, cabe destacar que las eventualidades que atravesó el AHPRN nos permiten resignificar el poder que conllevan los documentos -muchas veces en detrimento de memorias orales- en contraste con las condiciones históricas que permitieron su rescate o salvaguarda. Los Archivos silencian, omiten y olvidan a pesar de ser instituciones destinadas justamente a lo opuesto. Sin embargo, aquello que resguarda este Archivo es en el presente puesto en valor desde perspectivas muy diferentes a aquellas que lo fundaron. La triple función de los archivos nos invita a visualizar estas contradicciones que hacen a la institución. Los Archivos son garantes de derecho, memoria de Estado, y espacio de investigación.

Por último, queremos resaltar que la intención de hacer una historia del Archivo a partir de sus empleadas/ os se vincula con que la salvaguarda de la documentación para la construcción de la memoria histórica del pueblo, podemos pensarla ubicada en gestiones, conversaciones y relaciones entre empleados de diversas instituciones públicas. En todos estos años, el área de Cultura provincial de quien depende, nunca ha formalizado un organigrama que siente las bases para una permanencia y ordenamiento institucional acorde. Pero fundamentalmente tampoco se encuentra desarrollado el valor del Archivo en relación a sus funciones sociales y su respaldo a los derechos ciudadanos y humanos.

La voluntad y conciencia de algunos de sus trabajadores/as logró, en el cincuentenario de la creación de esta institución, mantener y resguardar gran parte del valioso material que se encuentra en el depósito, e incluso la gestión y mudanza al actual edificio en 2006. Este trabajo pretende resaltar que a pesar de las condiciones del mismo y de la escasa organización para su acceso a la población, en el presente el AHPRN ha sido fuente de numerosas investigaciones, juicios y recuperaciones de historias y biografías familiares. 
Post-scriptum: dedicamos este trabajo a Rebeca Pajón, incansable trabajadora del archivo, con quien compartimos muchas jornadas de trabajo y que accedió con el compromiso y afecto que la caracterizaba a contarnos sus historias del Archivo. Gracias Rebeca, Q.E.P.D.

\section{REFERENCIAS}

Binder, A. (2015). Del primer Congreso de Historia del Chubut y su trasfondo político y económico (Noviembre de 1967), Historia Regional, 33(12), pp. 151-169. Recuperado de: http://historiaregional.org/ojs/index.php/hist oriaregional/article/view/20/18

Bohoslavsky, E. y Soprano, G. (comp.) (2010). Un Estado con rostro humano. Funcionarios e instituciones estatales en Argentina (de 1880 a la actualidad). Buenos Aires: Universidad Nacional de General Sarmiento, Prometeo Libros.

Camino Vela, F. (2011). La dinámica política en la Provincia de Río Negro (Argentina) desde mediados del siglo XX: el predominio de la Unión Civica Radical. Tesis de doctorado. Sevilla: Universidad de Sevilla.

Cecchi, P. (2018). Sobre el alma del tehuelche puso el sello el historiador. La matriz represiva y militar en la construcción de las primeras instituciones historiográficas en Viedma, Actas del Primer Congreso de Historia de la Antropología Argentina. Buenos Aires: Instituto Nacional de Antropología y Pensamiento Social, Archivo Nacional de la Memoria, pp. 121-130. Recuperado de: https://journals.openedition.org/corpusarchivos/3798 ?file $=1$

Comaroff, J. y Comaroff, J. (1992). Ethnography and the Historical Imagination. Boulder: Westview Press.

Dall'armellina, M. y Cecilia Palma, C. (2018). Caciques y colonos en los márgenes del río Negro (1850-1950). Viedma: ESFA. Recuperado de: https://caciquesycolonos.com/

Derrida, J. (1994). Mal de Archivo. Una impresión freudiana. Recuperado de: https://redaprenderycambiar.com.ar/d errida/textos/mal+de+archivo.htm

Foucault, M. (2001). La Arqueología del saber. Buenos Aires: Siglo XXI.

García, N. (2006). De la naturaleza y del origen de la "neuquinidad". La institucionalización del pasado. Neuquén 1953-1976, Historia Regional, (24), pp. 11-27.

Heredia Herrera, A. (1986). Archivistica General. Teoria y Práctica. Sevilla: Diputación Provincial.

Heredia Herrera, A. (2020). La Nueva normalidad: relaciones entre el ayer inmediato y el futuro presente, Asociación Latinoamericana de Archivos. Recuperado de: https://www.alaarchivos.org/wp-content/uploads/2020/06/An tonia-Heredia.pdf

Kropff, L., Pérez, P., Cañuqueo, L. y Wallace, J. (comps.) (2019). La tierra de los otros. La dimensión territorial del genocidio indigena y sus efectos en el presente. Viedma: Editorial UNRN.

Nazar, M. y Pak Linares, A. (2007). El hilo de Ariadna, Politicas de la Memoria, (6/7), pp. 212-218.

Palma, C. (En prensa). Políticas oficiales del recuerdo: el Centro de Investigaciones Científicas de Río Negro. En P. Pérez (comp.), El papel del Archivo. Los archivos de la nor-Patagonia. Viedma: Editorial UNRN.

Pérez Morando, H. (2005). Estudios históricos en Río Negro, Diario Río Negro, 6 de diciembre. Recuperado de: htt p://www1.rionegro.com.ar/arch200512/06/o06a03.php

Pérez, P. (2016). Estado, indígenas y violencia en Patagonia Central 1878-1941. Buenos Aires: Prometeo.

Pérez, P. y Delrío, W. (2019). Cambios y continuidades en las (des)territorializaciones estatales en Río Negro (1878-1955). En: L. Kropff, P. Pérez, L. Cañuqueo y J. Wallace (eds.), La tierra de los otros. La dimensión territorial del genocidio indígena y sus efectos en el presente (pp. 31-70). Viedma: Editorial UNRN.

Popular memory group (1982). Popular memory: theory politics, method. En R. Johnson, G. McLennan, B. Schwartz, y D. Sutton (eds.). Making Histories. Studies in history writing andpolitics (pp. 205-252). Minneapolis: University of Minnesota Press.

Trouillot, M.R. (1995). Silencing de Past. Power and the Production of History. Boston: Beacon Press. 


\section{Notas}

1 Desde este proyecto de investigación PI UNRN 40 B 731 hemos llevado a cabo una campaña de spots radiales de visibilización del Archivo Histórico Provincial a partir de compilar experiencias de investigadores, realizadores y comunicadores, pobladores, comunidades indígenas y ciudadanía en general. Los spots fueron transmitidos por meses en radios de toda la Provincia y pueden ser escuchados en: https://soundcloud.com/proyecto-investigacion/sets/cam pana-archivos-e-identidad

2 La Patagonia tuvo el estatus diferencial de "Territorios Nacionales" desde 1884 hasta mediados de la década de 1950 cuando ingresó a la vida política y civil del resto de la nación argentina. En esos setenta años los habitantes de los territorios tenían un carácter subalterno de ciudadanía que les denegaba derechos civiles y políticos. Por esto, las autoridades de los Territorios -gobernadores, jefes de policía y jueces letrados- eran elegidos por el Poder Ejecutivo Nacional. Mientras que la población de los Territorios carecía tanto de la posibilidad del voto para elecciones del poder ejecutivo como de la representación en las cámaras de Diputados y Senadores.

3 Baste recordar la célebre frase del himno provincial, escrito por el padre Raúl Entraigas, "sobre el alma del tehuelche puso el sello el español”. La población auto-reconocida mapuche-tehuelche en el último censo nacional era de un $8 \%$. Sin embargo, sabemos que producto de la discriminación histórica muchas personas prefieren negar su identidad frente a los encuestadores.

4 El padre Entraigas, oriundo de Viedma fue autor de numerosas publicaciones salesianas sobre historia de la región (y en diarios como La Nación u otros de alcance regional). Era miembro de la Academia Nacional de la Historia.

5 Decreto 611 del 27/6/1967, Jornadas Anuales de Historia Rionegrina", Viedma; Resolución 480/67 del Ministerio de Asuntos Sociales, formación de la Junta de Investigaciones y Estudios Históricos de la Provincia de Río Negro; decreto modificatorio 669/68, Primer Congreso de Historia, sede Bariloche. El Segundo Congreso tuvo por sede a General Roca, en 1970; en 1972, a Cipolletti, y a Choele Choel en 1974.

6 Una lista más extensa presenta Pérez Morando en su nota "Estudios históricos en Río Negro": "Recordamos haber concurrido a aquel primer Congreso, como interesados en el pasado patagónico, al que fueron invitados destacados estudiosos y académicos de la historia: Paesa, Martínez, Barba, Furlong, Entraigas, Molinari, Vignati, Braun Menéndez, Martínez, Etchepareborda y Piccirilli, cuya comisión ejecutiva presidiera Entraigas, Héctor Daniel Rey como secretario; Nilo Juan Fulvi, coordinador general, y Omar A. Fossati, director provincial de Cultura, entre otros. Expusieron García Enciso, Arko, Dumrauf, Casamiquela, Furlong, Alvarez, Vignati, González Lonziéme, Biedma y Entraigas. Sin duda, en corto tiempo se había avanzado muchísimo en el propósito de conocer bien el pasado rionegrino que iniciaran Falkner, Viedma, Villarino y otros" (2005).

7 Testimonio de Nilo Fulvi, profesor de historia, Director del AHPRN entre 1967-1984, cuando asume como Ministro de Educación de la Provincia. Entrevista realizada en 2019 en la ciudad de Viedma.

8 Fulvi da dos ejemplos. El caso de la explotación de la vieira en el Golfo San Matías y la distribución de la tierra en Cubanea.

9 Rebeca Pajón relata que, así como muchos documentos fueron robados durante la dictadura, le consta que otros tantos fueron resguardados por empleados y luego devueltos al archivo.

10 Testimonio de Jorge Entraigas, profesor de historia, trabajador del AHPRN entre 1972-1990. Entrevista realizada en 2019 en la ciudad de Viedma.

11 Beatriz Moldes es antropóloga, docente y fue trabajadora del CIC es una de las profesionales que se radican en la zona a partir de esta propuesta.

12 El Consejo de desarrollo de Comunidades Indígenas es un organismo estatal para el cual se listaron los expedientes que se encontraban archivados como "Problemas Indígenas", provenientes de la Dirección de Tierras. A partir de esta sistematización, algunos casos olvidados, cajoneados o desvinculados de sus fondos fueron motor de demandas por derechos de un sector postergado de la sociedad rionegrina.

13 Joe Lewis es un destacado empresario inglés con acciones mayoritarias en empresas de energía como Pampa Energy hasta propietario de un club de futbol en Inglaterra (Tottenham Hot Spurs) que tiene una cantidad que excede los límites de la ley en la zona cordilerrana de Río Negro. Incluso cuenta con un fallo adverso en la justicia rionegrina para abrir los caminos que conducen al Lago Escondido, que hoy es de su propiedad, aun contra la legislación de nuestro país.

14 Testimonio de Rebeca Pajón, profesora de historia, trabajadora del AHPRN entre 1995-2015. Entrevista realizada en 2019 en la ciudad de Viedma.

15 Según Fulvi, este vínculo se correspondía también con un cambio en la política de la Academia que buscó organizar encuentros y jornadas por fuera de Buenos Aires.

16 Durante el 2010, Cecilia Palma entrevistó en Viedma a Antonia Peronja, Eleonora Nervi, Alicia Pulita, Ramón Varela y Beatriz Moldes. Todos trabajadores del CIC y en estrecha relación al AHPRN. 
17 Este fondo documental fue cedido por Justicia con una serie de descriptores que listan los expedientes que fueron transferidos. La descripción no está completa pero abarca las primeras décadas del siglo XX.

18 Contemporáneamente se reunía también el material para los Museos de Viedma, Gral. Roca y Valcheta, con los resultados de las campañas del CIC. en este contexto destaca Fulvi se ingresaron los esqueletos extraídos del IDEVI por Casamiquela.

19 Testimonio de Beatriz Moldes, licenciada en antropología, trabajadora del CIC que se radica en la zona a partir del proyecto del Centro. Entrevista realizada en 2019 en la ciudad de Viedma.

20 Juan Mario Raone, militar de carrera (suboficial retirado del ejército argentino), asesor político del Movimiento Popular Neuquino en la Legislatura, historiador regional, miembro de la Academia Nacional de la Historia; cotidiano colaborador en las salas y depósitos desde los inicios del CIC.

21 Podemos mencionar la existencia de Ley Provincial A 722/2007: Archivo General de la Provincia de Rio Negro. Así como la Ley Provincial 4706/2011 que determina la creación del Archivo Histórico Digital Provincial (AHDIP) y también la Ley F 3656/2007 de Protección y conservación del Patrimonio Cultural de la Provincia de Rio Negro. Sin embargo, como dijera Antonia Heredia en una conferencia reciente “...hemos pasado, al menos en España, de una ausencia de legislación específica a una sobredosis de leyes y de normas que muchas veces, dado su número, es imposible leer, conocer y por tanto difícil de aplicar...”. Por esto, queremos aclarar que, si bien existe legislación al presente, su mera existencia no tiene influencia sobre los avatares del AHPRN. 